\title{
Surgery of the glaucomas
}

\author{
P. G. WATSON \\ Cambridge
}

Once the decision to operate has been made, one question remains: which surgical procedure is best for that patient? As time has passed the choice of procedure has become greater and the reasons for choosing a particular operation have now become very precise.

Historically there have been three periods when there has been a surge of interest in the surgical treatment of glaucoma. Each period has corresponded with a change of thought on the aetiology of the condition or with improvement in instrumentation, and has been followed by a long period in which the results have been critically assessed. As a result, certain procedures have stood the test of time whilst others have been rejected, either because of poor results, or because the surgical procedure was too hazardous, difficult, or time-consuming.

The first attempts at surgical treatment of glaucoma were made by Mackenzie (1830) and in 1835 by Middlemore by paracentesis and scleral puncture, but the first successful surgical procedure was von Graefe's iridectomy (1857b). In the next year Critchett (1858) developed the operation of irodesis-a type of iris inclusion.

As it became obvious that these operations did not control all types of glaucoma, there followed another series of attempts to improve the lot of the patient with chronic simple glaucoma. The earliest of these were made by de Wecker with his anterior sclerotomy and by Argyll Robertson (1876) who trephined behind the ciliary body. There followed a period of assessment and then came the operations of anterior sclerotomy by Herbert (1903), partial sclerotomy and iridectomy by Lagrange (1906), cyclodialysis by Heine (1906), iridencleisis by Holth (1907), and the corneo-scleral trephine of Elliot (1909), which were all devised within the first decade of the 2oth century and have survived till the present day.

The only significant advances from i 9 Io until the advent of the operating microscope were the peripheral iridectomy of Curran (1920), the goniotomy of Barkan (1938), and more recently the iridectomy and cautery of Scheie (1958). The surgical microscope has revived interest in glaucoma surgery, for it has now become possible to attempt surgery of the trabeculum if this indeed is where the pathology in open-angle glaucoma exists. In recent years, therefore, we have seen the trabeculotomy of Redmond Smith (1950) and Allen and Burian ( 1962 ), the sinusotomy of Krasnov (1964), the trabeculotomy of Harms (1966) and Harms and Dannheim (1970) and the trabeculectomy of Cairns (1968).

A good surgical operation must be judged by the following criteria:

(I) The diseased or damaged tissue must be removed without detriment to the organ involved.

(2) The operation must cure or control the condition for which it is performed.

(3) The surgeon must be able to see the tips of his instruments at all stages of the operation so that the operation remains under his control at all times.

(4) The procedure must be free of operative complications which cannot be readily contained.

(5) The operation must be free of all major postoperative complications.

(6) The procedure should be as simple as possible, so that there may be an economy of effort and time. 
If any of these criteria are not fulfilled then a further search for a better procedure is justified, but if one operation replaces another it must be significantly better than its predecessor.

Peripheral iridectomy by the $a b$ externo approach is one of the best examples of a good operation.

SURGERY FOR OPEN-ANGLE GLAUCOMA

The logical surgical treatment of this condition still awaits the certainty of the cause. In the meantime we assume that the blockage is at the trabecular meshwork or just beyond it and our efforts, as we have seen, have been directed to relieving this site of blockade. Blockage in the region of the trabecular meshwork certainly exists in glaucoma whether it be the cause or the effect of the condition, so it is reasonable that efforts to relieve the blockage have been blessed by some degree of success.

Assuming that surgery has become necessary because of inability to tolerate or failure of control by medical therapy, what is the best operation for the control of the intraocular pressure? We have seen the numerous choices open to us and we have discussed what constitutes a good procedure. Most of those available to us fall down in some respect of these criteria, e.g. the Elliot's trephine may fail because the tip of the instrument cannot be properly visualized, giving rise to serious operative and postoperative complications (Leydhecker, I 967). Sclerotomy using a punch is less dangerous, but the instruments are too large and the distortion of the eye too great. Iridencleisis unnecessarily destroys the integrity of the pupil. Cyclodialysis attacks the ciliary body and has a relatively poor success rate (Leydhecker, 1966b). Implantation of foreign bodies in the eye are rarely successful and should be avoided if other procedures are available. The procedures least open to objection are the Scheie operation and the Cairns type of trabeculectomy.

At the present time the first operation for patients with open-angle glaucoma should be a modification of the Cairns type of trabeculectomy (Watson, 1970). Briefly, this operation is carried out through the operating microscope.

A superficial flap $5 \times 6 \mathrm{~mm}$. is fashioned through two-thirds the depth of the sclera, conjunctiva and Tenon's capsule having been reflected in the usual manner (Fig. I). When this flap is retracted with a suture the scleral spur can be seen through the semi-transparent sclera (Fig. 2).

(I)

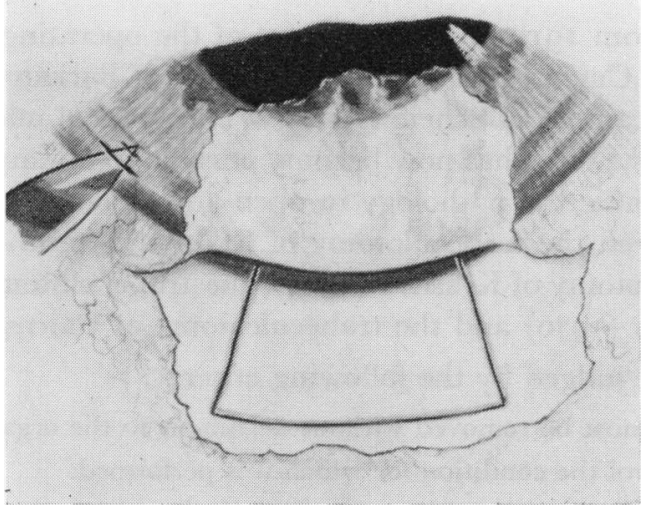

(2)

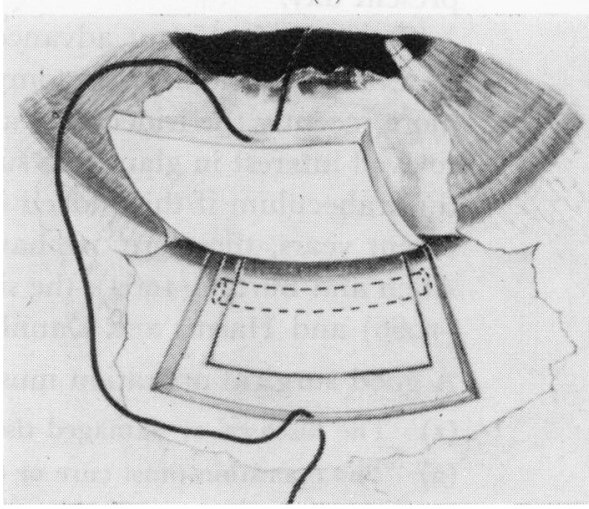

FIG. I Paracentesis at the limbus with the tip of the needle just penetrating the anterior chamber. The superficial scleral flap is fashioned $\frac{2}{3}$ thickness of sclera, $5 \mathrm{~mm}$. radially, and $6 \mathrm{~mm}$. circumferentially

FIG. 2 The superficial flap dissected forwards just into corneal tissue. A suture is placed in the superficial flap to aid retraction and keep the anterior chamber closed. The scleral spur is visible through the deep lamellae. The deep flap is fashioned through the full thickness of the sclera behind the scleral spur 
A deep flap is then dissected down to the ciliary body and the scleral spur, which is firmly attached to the underlying structures, is dissected free. The incisions are now extended forwards (Fig. 3) and the flap which now contains trabeculum is excised with Vannas scissors (Figs 4 and 5). A peripheral iridectomy is performed and the superficial flap is sutured back into place.

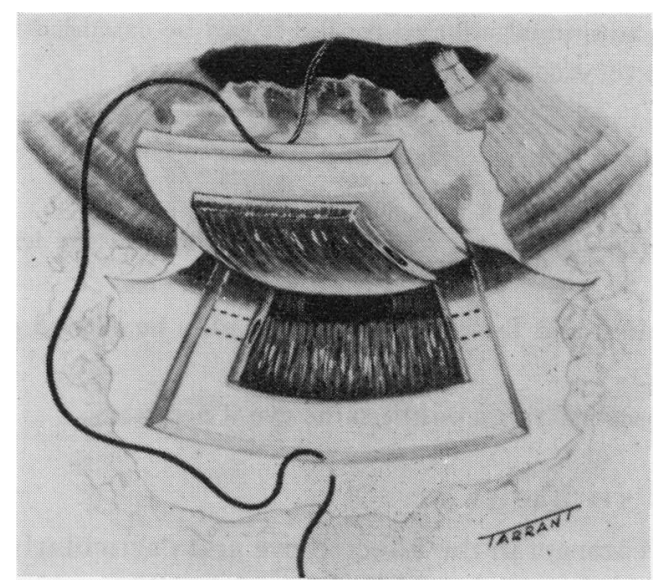

FIG. 3 The deep flap containing scleral spur, Schlemm's canal, and trabecular tissue is dissected forwards before excision

The choroid and iris are different colours and easily distinguished. The scleral spur is firmly attached to underlying choroid and has to be dissected free

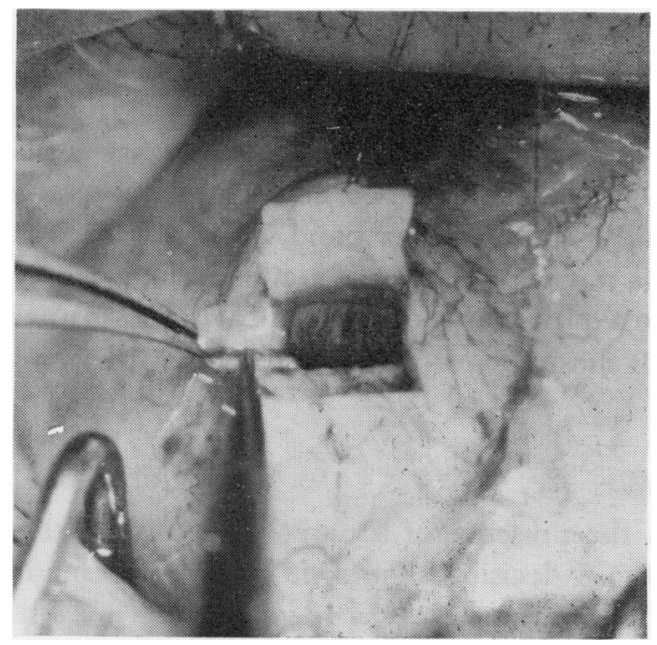

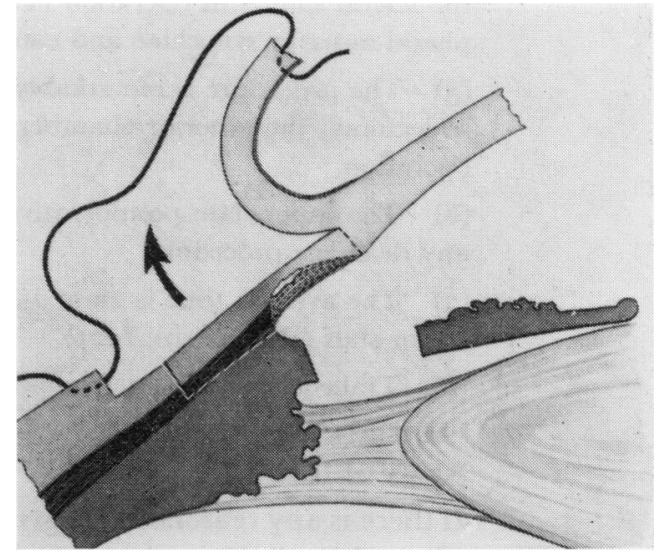

FIG. 4 Block of tissue removed at operation

F I G. 5 Deep flap folded back to show from behind forwards: the scleral spur, the pigmented ciliary band, the trabecular tissue, and the cornea

The anterior chamber is still present. The peripheral iridectomy can be performed before or after the excision of the deep flap

This operation is to be preferred for several reasons:

(I) It removes an area of damaged trabeculum.

(2) The resuturing of the superficial flap restores the eye almost to its preoperative state. I find this much easier than the direct approaches to the canal of Schlemm as in sinusotomy and Harms's trabeculotomy. I have had poor results from the few I have attempted but this could be due to lack of experience. 
(3) Control of the glaucoma without further medication or operation is achieved in 90 per cent. of patients. In 85 per cent. control is achieved by subconjunctival drainage, but in the majority the blebs are diffuse and often barely visible.

(4) The operation performed under the surgical microscope is at all times under the control of the surgeon and the tips of the instruments can always be seen. Because the incision is made behind the scleral spur, this operation has the additional advantage that it can be extended to divide peri- $\frac{}{2}$ pheral anterior synechiae and can also be used in cases of aphakic glaucoma.

(5) The procedure is remarkably free from operative complications; in particular, as in peripheral in iridectomy, the anterior chamber is never completely lost and is always left full at the end of the $\vec{O}$ operation.

(6) The immediate postoperative complications are few, but late changes in the lens occur as in any drainage procedure.

(7) The average time is 20 to 25 minutes and the procedure can readily be carried out by trained junior staff (Rubinstein, 1971).

(8) Trabeculectomy can be repeated several times on the same eye if necessary.

REDUGED VISUAL AGUITY DUE TO GATARAGT

If there is any reasonable degree of cataract in the affected eye and particularly if the lens is affected in the opposite eye, then the lenses should be removed. There is evidence that all cataract sections drain after operation and in the presence of open-angle glaucoma some drain permanently. In many cases it is true that the control of intraocular pressure may only last for 6 months to a year, but these eyes can be subjected to further operation if the pressure is not controlled by miotics. This is certainly preferable to combined drainage and cataract procedures from which I have seen two cases of epithelial downgrowth.

SURGERY OF PRIMARY CLOSED-ANGLE GLAUCOMA

Observation of a very narrow angle without symptoms

It has been shown by Törnquist (1958) that if the depth of the anterior chamber is less than $1.5 \mathrm{~mm}$. the chances of developing closed-angle glaucoma are $52: \mathrm{I}$. Anterior chambers deeper than this but with peripheral irido-corneal contact also have a very high $:$ chance of developing an acute attack. On the other hand, good though it is as a surgical procedure, peripheral iridectomy is not entirely free of complications. My preference is $ᄋ$ therefore to subject these patients to a dark room and/or mydriatic test, to operate if there is any doubt, and to warn the rest of the patients of the symptoms which may indicate an attack.

Symptoms suggestive of closed-angle attacks

Once symptoms have occurred in the presence of a narrow angle then the operation of peripheral iridectomy is indicated. A decision should, if possible, be made without the use of provocative tests. Once intermittent attacks have become established, miotics will not abolish them for long, and may even induce an acute attack by drawing the $\underset{\Phi}{\mathbb{C}}$ peripheral iris into the angle. Operation is therefore the treatment of choice and only $\stackrel{\mathcal{O}}{?}$ age and infirmity or refusal of operation should alter this decision.

The acute closed-angle attack

Surgical treatment during the acute stage of the condition is now rarely necessary Adequate therapy with Pilocarpine and osmotic agents almost always reduces the pressure 
to reasonable limits within a few hours of the start of therapy. What then is the correct time to operate and what procedure should be undertaken?

(a) Control with miotics There is no immediate urgency for surgery if the pupil is circular and miotic and the pressure returns to normal within a few hours, remaining controlled with miotics alone. If possible at least a week should elapse before surgery and miotic therapy should be supplemented with steroids if there is any sign of anterior chamber inflammation. After this time the eye is quiet and not congested and is readily treatable by peripheral iridectomy.

(b) If the pressure returns to normal and is controlled with miotics but the pupil remains eccentric, then the same routine is followed but the peripheral iridectomy is performed at the site of the infarcted iris, and it is made large enough to prevent the flaccid iris from rolling back into the angle.

(c) Control only with osmotic agents If the pressure needs to be controlled with osmotic agents in addition to miotics, then there is more urgency for operation. Peripheral iridectomy is performed if the pressure starts to rise again or as soon as the anterior chamber is free of cells.

(d) Failure of control in spite of peripheral iridectomy The large majority of eyes will be controlled by peripheral iridectomy even though there may be very extensive peripheral anterior synechiae. A filtering operation, such as Scheie's procedure or an iridencleisis, has been suggested in the acute stage of the disease but my experience of these operations has been most unhappy. I have seen failure of the anterior chamber to re-form and one case of malignant glaucoma after drainage procedures. I have seen six cases of sympathetic ophthalmitis in my career, four of which have followed the use of iridencleisis during an acute attack of glaucoma. Because of these experiences I follow the suggestion of Shaffer (1958): do a peripheral iridectomy, wait, reassess the patient after an interval, and then if necessary proceed to a trabeculectomy later. I prefer this procedure as it can be extended to divide peripheral anterior synechiae under direct visual control from behind the scleral spur.

\section{GHRONIC GLOSED-ANGLE GLAUCOMA}

Again peripheral iridectomy will control many of these patients. Miotics in addition to peripheral iridectomy will control many more. There remain a few who require filtering surgery and here again trabeculectomy with peripheral iridectomy performed from behind the scleral spur, with or without the division of peripheral anterior synechiae, has a great deal to recommend it. This procedure has the additional advantage that the wound is sutured after excision of the trabecular area so that the dangers of late loss of the anterior chamber are much reduced.

\section{PLATEAU IRIS}

It is possible that this is one of the few remaining indications for the broad iridectomy of von Graefe. In the few cases of true plateau iris that I have encountered, my experience has been that of Étienne ( $1969 \mathrm{~b})$ - that peripheral iridectomy was ineffective and failed to relieve the pupil block even with the use of miotics.

\section{THE SECOND EYE}

The evidence is regrettably overwhelming that the second eye will develop an acute or sub-acute attack within 5 years of the first evidence of the disease in the opposite eye (Winter, 1955; Adams, 1955; Bain, 1957; Lowe, 1962). There is no doubt, therefore, that any person who has had an acute or sub-acute attack in one eye must have a peripheral iridectomy in the opposite eye as soon as possible after the first operation. This advice is almost always accepted if the proposition is made during the acute phase of the disease, 
but it is often less easy to persuade the patient after the first eye has been treated. The $\frac{\overline{3}}{3}$ only difficulties arise in the pre-glaucoma stage, but if the decision to do surgery on one eye is correct it must apply to both in a bilateral disease.

OTHER TYPES OF GLAUCOMA FOR WHICH SURGERY IS INDICATED

Although the majority of patients with glaucoma can be treated with either peripheral $\stackrel{\mathbb{\Phi}}{\varrho}$ iridectomy or trabeculectomy, repeated if necessary several times, there remain a few patients who will either not respond to this form of therapy or require a different type of $\vec{\circ}$ operation.

\section{Congenital glaucoma}

The treatment of congenital glaucoma has been discussed elsewhere (Rice, p. 294) but suffice it to say that goniotomy properly performed will control the majority of patients in infancy. If several goniotomies fail to control the intraocular pressures the problem becomes much more difficult. Approximately $5^{0}$ per cent. of these patients can be con- 근 trolled by a drainage procedure (Shaffer, and Weiss, 1970). Ectasia of the wound can occur particularly after trephine or sclerotomy operations and the zonule of a stretched eye $\stackrel{m}{\frac{3}{7}}$ is lax, predisposing to lens incarceration. Theoretically the most satisfactory procedure in the stretched eye should be the Harms trabeculotomy. If Schlemm's canal cannot be $\overrightarrow{0}$ identified then the incision can be deepened and very lightly cauterized as in Scheie's procedure.

In patients with Sturge-Weber syndrome and new vessels in the angle, the only alternative to medical therapy is cyclocryotherapy $\left(-70^{\circ}\right.$ for between 30 and 45 seconds for the whole circumference of the globe and perhaps repeated on two occasions or even more). This is a destructive operation and the chances of finding the happy medium between failure of control and complete destruction of the globe are slim indeed.

Juvenile glaucoma and glaucoma in the Negro eye

Failure of a drainage procedure in the older child or young adult whose sclera is of normal thickness is often due to the excess scarring in the thick episclera occluding the new drainage channels. In these patients a trabeculectomy should be performed as a first procedure. If this needs to be repeated, then all the episclera in the region of the wound is removed in the hope of reducing the contraction of this tissue. In addition, intensive steroid therapy is used after the operation. I have very little experience of glaucoma in the Negro $\frac{I}{O}$ eye, but have always assumed that the causes of failure are the same as those in the young person. Redmond Smith's trabeculotomy was designed to deal with this problem and o has met with success. The great advantage of this operation is that a very large area of trabeculum is incised and it should be performed if previous surgery has failed.

SECONDARY GLAUCOMAS

As time passes one hopes that all the glaucomas will be "secondary" in order that one can attack the cause of the condition. Surgery is directed at removal of the cause; angle blockage as in the pigmentary glaucomas, cataract extraction in the lens-induced glaucoma, removal or by-pass of peripheral anterior synechiae or pupil block. If surgery is necessary for any other reason then one is coming very close to the end of the road. There are, however, one or two procedures which merit attention under these circumstances. 


\section{Aphakic glaucoma}

Because of the dangers of vitreous incarceration in the wound after operation, cyclodialysis has been recommended. If the glaucoma is due to peripheral anterior synechiae these can in fact be better tackled through a trabeculectomy incision from behind the scleral spur as a modified cyclodialysis. If it is felt that vitreous is likely to present, then the trabeculectomy is not completed. If, however, pupil block is thought to be a factor, then a peripheral iridectomy can be performed through the same incision. If it is felt that persisting simple glaucoma is contributing to the aphakic glaucoma, then a section of trabeculum can be excised in addition.

Aniridia, essential iris atrophy, exceptionally wide peripheral anterior synechiae, and some Negro and juvenile patients on whom no other operation has been successful

In these circumstances the operation of iridocyclo-retraction (Krasnov, 1971) seems to be ideal, as it keeps the angle open and away from the iris in conditions in which it is impossible to achieve this by any other procedure. At present I would not recommend this in young eyes except as a last resort, because of the severe ectasia which it appears to produce at the site of operation.

\section{Haemorrhagic glaucoma}

I think I have had the same dismal experience as everyone else with these eyes and use the same destructive procedures such as cyclocryotherapy. Lowering the pressure by any other means in an eye which is not painful seems to allow the neovascularization to increase, leading to a further downhill course and almost inevitable to enucleation. If it seems essential to do an intraocular operation, Scheie's procedure has the greatest chance of success.

The patient usually has to pay a price for surgery in the form of altered or changing vision or worse (Frühauf, Müller, and Sismuth, 1967). Surgery for glaucoma should never be undertaken except when other methods of treatment have failed or have been shown to be less effective. Should surgery become necessary, however, I hope this paper will give some guidance to its use.

\section{COMMENTARY}

\section{TRABEGULEGTOMY}

Professor Richardson discussed the hundred trabeculectomies which he had performed. He felt that it was most certainly a filtering procedure and those cases which did not filter he assumed to work as a cyclodialysis. The operation had no broader spectrum of success than other filtering procedures but it had one real advantage, the lack of immediate postoperative flat anterior chambers. In the hundred cases that he had performed he had had to re-form the anterior chamber only twice and had flat anterior chambers during the first 3 days in only six or seven patients. He also felt that if a trabeculectomy failed then the eye was not significantly disturbed as far as the external anatomy was concerned, so that several procedures might be repeated close to each other should this be necessary.

Dr. Simmons discussed the method which he and Dr. Chandler had been using for the past 7 years in which a scleral contact lens was placed over the bleb from a standard drainage procedure in order to reduce the excess outflow from the scleral opening during the early days and weeks after surgery. When the eye had recovered from the surgical insult and choroidal separation was no longer a problem, the contact lens was removed, allowing the eye to release aqueous gently without 
development of hypotony. He had found that this had greatly reduced the incidence of flat anterior chamber and choroidal separation and also gave a very diffuse bleb. Tension control over a prolonged period also seemed to be very much better than with conventional operations, probably because the aqueous was spread out over a broad area, preventing adhesion between the conjunctiva and underlying tissue, so that the bleb did not become localized there during the early stages following surgery.

Dr. Simmons questioned whether trabeculectomy or other microsurgery might be dangerous in the hands of a surgeon who undertook glaucoma surgery only occcasionally. Dr. Anderson expressed the opinion that recently trained surgeons who used a microscope routinely (e.g. for cataract surgery) would find trabecular surgery easy and safe, but he agreed that these were difficult operations for surgeons who did not use the operating microscope frequently.

\title{
Summary
}

\author{
PROFESSOR HANS GOLDMANN
}

Berne, Switzerland

Certain points from this Symposium are particularly memorable. The first concerns the changes which occur in glaucomatous atrophy of the disc. They are characterized by the combined disappearance of nerve fibres and glial tissue in contradistinction to simple atrophy in which only the nerve fibres disappear. Paradoxically, in juvenile glaucoma, restoration of pressure, not hypotony, may restore the normal disc appearance, a phenomenon not yet understood. There is no doubt that there is a pressure-dependent circulation sensitivity which is different in different eye tissues. The circulation at the disc and its surroundings shows the greatest sensitivity to a rise in pressure which differs in different sections of the disc and of the adjacent uveal tissue. The rest of the uvea is less sensitive and the retina still less so. The significance of the fluorescein appearance-time of the disc is still a matter of controversy. The influence on function when the papillary circulation is damaged is particularly impressive in cases of true low tension glaucoma in which general vascular and blood factors have been shown to play an important part. The axonal flow in the nerve fibres may be of some importance in the development of nerve fibre degeneration and should be further examined.

The indication is becoming stronger that, at least in the majority of cases, the obstruction to aqueous flow lies between the anterior chamber and Schlemm's canal. There is still uncertainty about the role of the trabecular meshwork and the canal endothelium. The finding of intracellular vacuoles and canals in the endothelium poses new problems. Is active transport involved? The seemingly good relationship between pressure-head and flow speaks rather against it. In patients with simple glaucoma the number of vacuoles is diminished. This may be due to a pathological state of the endothelial cells or to a diminished supply of aqueous, or it may be an artefact caused by therapy. When hypertension follows trauma the changes in the trabecular meshwork are the cause of the high pressure, even though there may be other structural changes in the eye such as angle recession. 